\title{
44549 - RIGHT-HEART VEGETATION PENETRATING THROUGH VENTRICULAR SEPTAL DEFECT IN PATIENT WITH INFECTIVE ENDOCARDITIS
}

\author{
Nasr Hegazy, Toronto General Hospital, University Health Network, Toronto, ON, \\ Canada; \\ George Djaiani, Toronto General Hospital, University Health Network; \\ Glen Van Arsdell, Toronto General Hospital, University Health Network; \\ Jacek Karski, Toronto General Hospital, University Health Network;
}

PURPOSE: Infective endocarditis is one of the most devastating manifestations of staphylococcus bacteremia (1). Right -heart vegetations with the ventricular septal defect (VSD) are rare. We describe a rare case of endocarditis with mobile vegetation originating in the rightheart and penetration through VSD into the left-heart.

CLINICAL FEATURES: After obtaining patient consent, we describe a 26year- old male with a past medical history of corrected VSD at the age of 2, and presented with the signs of bacterial endocarditis following a routine dental procedure. Blood culture revealed a considerable staphylococcus growth and the intravenous antibiotic treatment was commenced.

Transesophageal echocardiography (TEE) revealed a VSD with mobile vegetations, as well as aortic insufficiency and normal biventricular function. <Figure 1>

The patient was scheduled for emergency surgery. A combination of moderate dose opioids and inhalational anesthesia was used. Standard management of tepid cardiopulmonary bypass was employed. During surgery, the preoperative TEE findings were confirmed .The surgery involved a debridement of vegetations, closure of VSD and repair of aortic valve. Separation from CPB was uneventful. Postoperative treatment included administration of cloxacillin and gentamicin for 6 weeks. The patient was discharged from the hospital on the 10th postoperative day without any end-organ morbidity.

CONCLUSION: Early clinical diagnosis of infective endocarditis with a TEE confirmation is paramount in successful outcome in this high-risk cardiac surgery.

REFERENCES: 1) Murray RJ. Staphylococcus aureus infective endocarditis: diagnosis and management guidelines. Intern Med J 2005; 35(2): s25-s44. 